\title{
Experimental Evidence for Epitaxial Silicene on Diboride Thin Films
}

\author{
Antoine Fleurence, ${ }^{1}$ Rainer Friedlein, ${ }^{1}$ Taisuke Ozaki, ${ }^{1,2}$ Hiroyuki Kawai, ${ }^{1}$ Ying Wang, ${ }^{1}$ and Yukiko Yamada-Takamura ${ }^{1, *}$ \\ ${ }^{1}$ School of Materials Science, Japan Advanced Institute of Science and Technology (JAIST), \\ 1-1 Asahidai, Nomi, Ishikawa 923-1292, Japan \\ ${ }^{2}$ Research Center for Simulation Science, Japan Advanced Institute of Science and Technology (JAIST), \\ 1-1 Asahidai, Nomi, Ishikawa 923-1292, Japan \\ (Received 14 February 2012; published 11 June 2012)
}

\begin{abstract}
As the Si counterpart of graphene, silicene may be defined as an at least partially $s p^{2}$-hybridized, atomthick honeycomb layer of Si that possesses $\pi$-electronic bands. Here we show that two-dimensional, epitaxial silicene forms through surface segregation on zirconium diboride thin films grown on Si wafers. A particular buckling of silicene induced by the epitaxial relationship with the diboride surface leads to a direct $\pi$-electronic band gap at the $\Gamma$ point. These results demonstrate that the buckling and thus the electronic properties of silicene are modified by epitaxial strain.
\end{abstract}

DOI: 10.1103/PhysRevLett.108.245501

While silicon is right below carbon in the periodic table of elements, valence orbitals do not $s p^{2}$ hybridize as easily as for its smaller counterpart. Although it is difficult to form, freestanding silicene has been predicted to be stable [1-4]. Astonishing electronic properties, such as the presence of a Dirac cone, are expected to prevail even in its predicted, slightly-buckled form where neighboring $\mathrm{Si}$ atoms are displaced out of plane [1-4]. Because it can be easily buckled, silicene may exist with a variety of lattice constants, atomistic structures and with a varying $s p^{2} / s p^{3}$ ratio. In contrast to graphene, this structural flexibility may give rise to altered electronic properties, which no one has imagined so far.

While freestanding silicene could not be produced up to now, experimental evidence even for the existence of epitaxial silicene is rather limited but does exist. Honeycomblike Si structures occur in disilicides [5-8]. Recently, silicene ribbons obtained by deposition of silicon atoms on the $A g(110)$ surface have been well characterized [9-12]. On the other hand, evidence for the formation of epitaxial silicene sheets on $\operatorname{Ag}(111)$ based on scanning tunneling microscopy (STM) images alone [13] may be insufficient.

In this Letter, we demonstrate that epitaxial, twodimensional silicene forms spontaneously on (0001)oriented thin films of zirconium diboride $\left(\mathrm{ZrB}_{2}\right)$, a conductive ceramic, that are grown epitaxially on $\mathrm{Si}(111)$ wafers. Through a detailed characterization of the structure and electronic properties, we further show that a particular buckling induced by the epitaxial relationship with the diboride surface causes the opening of a direct $\pi$-electronic band gap. While graphene is robust in terms of its crystal structure, our results show that silicene is

Published by the American Physical Society under the terms of the Creative Commons Attribution 3.0 License. Further distribution of this work must maintain attribution to the author(s) and the published article's title, journal citation, and DOI.
PACS numbers: 61.46. $-\mathrm{w}$, 68.37.Ef, 73.22.-f, 81.05.Zx

more flexible which allows an engineering of the band structure through epitaxy.

Thin $\mathrm{ZrB}_{2}$ films were grown by ultrahigh vacuum (UHV) chemical vapor epitaxy [14]. Prior to each experiment in separate UHV setups, native oxide was removed by annealing at 750 to $800{ }^{\circ} \mathrm{C}$ for ten hours under UHV conditions $[14,15]$. For STM, chemically etched Pt-Ir tips were used. Si $2 p$ photoelectron spectra were recorded at BL-18A of the KEK-PF synchrotron radiation facility (Tsukuba, Japan), using third-order light $(h \nu=130 \mathrm{eV})$. Angle-resolved ultraviolet photoelectron spectroscopy (ARUPS) was carried out in a home-based setup using He I light $(h \nu=21.2 \mathrm{eV})$ [16] with the samples held at approximately $140 \mathrm{~K}$. The total energy resolution was better than 130 and $30 \mathrm{meV}$, for the two setups, respectively. The density functional theory (DFT) calculations within a generalized gradient approximation [17] were performed by the openmX code $[18,19]$ which is based on norm-conserving pseudopotentials generated with multi reference energies and optimized pseudoatomic basis functions. The slab consisted of seven $\mathrm{Zr}$ and six $\mathrm{B}$ layers composed of the experimentally determined $(2 \times 2)$ unit cell (UC) of $\mathrm{ZrB}_{2}(0001)$ and were terminated by Si layers on both sides. The structure was fully optimized until the maximum force became less than $10^{-4}$ Hartree/Bohr. Core-level energy differences were calculated within the framework of noncollinear DFT including spin-orbit coupling using fully relativistic pseudopotentials where the $\mathrm{Si}$ $2 p$ states were explicitly treated as valence states.

Evidence for a honeycomb structure.-As discussed previously [14,15], the oxide-free single-crystalline $\mathrm{ZrB}_{2}(0001)$ thin film surface consists of a few hundred nanometer-wide terraces exhibiting a $(2 \times 2)$ reconstruction specific to the films grown on silicon [14]. Since the hexagonal-close-packed $\mathrm{Zr}$ layer at the surface is intact [14], the $(2 \times 2)$ reconstruction may be related to ad-atoms most likely segregating from the Si substrate. 
Figure 1(a) shows a large-scale STM image. Stripes with domain boundaries run along $\langle 11 \overline{2} 0\rangle$ directions. These domains are offset with respect to each other toward $\langle 11 \overline{2} 0\rangle$ directions that differ from those of the orientation of the domain boundaries. The repetition of the spacing between boundaries and the alternation of the direction of the offsets are signature of the spontaneous formation of stress domains [20] as a result of large-scale interactions within a two-dimensional layer of these ad-atoms. The constant-current STM image in Fig. 1(b) reveals fine details of this layer, which correspond to a honeycomb mesh with the lattice constant of approximately $3.65 \AA$. The layer is compressed by $5 \%$ with respect to a bulk $\mathrm{Si}(111)$ bi-layer with the in-plane epitaxial relationship of $\mathrm{Si}[11 \overline{2}] \| \mathrm{ZrB}_{2}[11 \overline{2} 0]$ such that its $(\sqrt{3} \times \sqrt{3}) \mathrm{UC}$ is adjusted to that of the $\mathrm{ZrB}_{2}(0001)-(2 \times 2) \mathrm{UC}$.

Atomic-scale buckling of a Si layer-Proof for the presence of Si atoms at the surface comes from the surfacesensitive Si $2 p$ photoelectron spectrum obtained at normal emission, shown in Fig. 2(a). The sharpness of the features is a clear indication of $\mathrm{Si}$ atoms in well-defined chemical environments different from those at the $\operatorname{Si}(111)-(7 \times 7)$ surface [21]. As quantified by a peak fitting [22], the Si $2 p$ doublet consists of three components labeled $\alpha, \beta$, and $\gamma$, which are related to three different chemical environments of the $\mathrm{Si}$ ad-atoms, denoted $\mathrm{Si}_{A}, \mathrm{Si}_{B}$, and $\mathrm{Si}_{C}$. Relative to the main peaks which have major contributions from the $\beta$ chemical state, $\gamma$ and $\alpha$ are shifted $140 \pm 5 \mathrm{meV}$ and $260 \pm 5 \mathrm{meV}$, respectively. The respective areal weights for peaks $\alpha, \beta$, and $\gamma$ are $0.32 \pm 0.05,0.55 \pm 0.05$ and $0.13 \pm 0.05$. The single in-plane position of a Si honeycomb structure matching the ratio between atomic sites
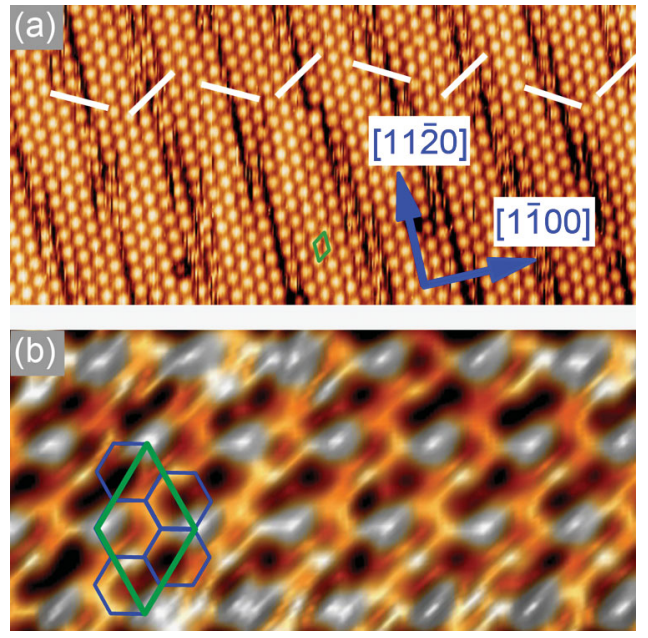

FIG. 1 (color). STM images of the $(2 \times 2)$-reconstructed $\mathrm{ZrB}_{2}(0001)$ surface with different length scales: (a) $20 \mathrm{~nm} \times$ $9.5 \mathrm{~nm}, I=55 \mathrm{pA}, V_{s}=700 \mathrm{mV}$, (b) $4.2 \mathrm{~nm} \times 2 \mathrm{~nm}, I=$ $600 \mathrm{pA}, V_{s}=100 \mathrm{mV}$. The white lines emphasize the direction of offsets between successive domains. The $(2 \times 2) \mathrm{UC}$ and the honeycomb mesh are emphasized by green and blue solid lines, respectively. derived from the spectrum within the error bars is presented in Fig. 2(b). In the $\mathrm{ZrB}_{2}(0001)-(2 \times 2) \mathrm{UC}$, two $\mathrm{Si}_{A}$ atoms are sitting on hollow sites of the $\mathrm{Zr}$ lattice, three $\mathrm{Si}_{B}$ atoms are located at the intermediate position between top and bridge sites, and one $\mathrm{Si}_{C}$ atom is on the top of a $\mathrm{Zr}$ atom. This Si honeycomb structure may then be considered to be an atom-thick, epitaxial Si layer.

Geometrical information with regard to the height of the three components may be found by diffraction of $\mathrm{Si} 2 p$ photoelectrons [6,23]. As shown in Fig. 2(c), the $\alpha$ component maintains its intensity up to the polar emission angle of $\theta=60^{\circ}$ along the [11100] direction of the $\mathrm{ZrB}_{2}(0001)$ layer, while it decreases by about $30 \%$ with increasing $\theta$ along the [112̄0] direction. The latter reduction can qualitatively be attributed to diffraction of $\mathrm{Si}_{A}$ photoelectrons by $\mathrm{Si}_{B}$ atoms in the particular direction. It is thus understood that $\mathrm{Si}_{A}$ atoms are in a lower position compared to $\mathrm{Si}_{B}$ atoms proving atomic-scale buckling within the honeycomb Si layer. On the other hand, such an effect is not observed between $\mathrm{Si}_{B}$ and $\mathrm{Si}_{C}$ atoms. The result indicates that $\mathrm{Si}_{C}$ atoms are located at a height different from $\mathrm{Si}_{A}$ atoms while these two types of atoms form one of the two sublattices of the honeycomb structure: a $(\sqrt{3} \times \sqrt{3})$-reconstructed Si layer.

Band structure of the epitaxial Si layer-The Brillouin zones (BZs) of the $(2 \times 2)$-reconstructed $\mathrm{ZrB}_{2}(0001)$ surface, which corresponds to that of the $(\sqrt{3} \times \sqrt{3})$ reconstructed Si honeycomb layer, and the unreconstructed silicene are shown in Fig. 3(a). Note that the $K_{\mathrm{Si}}$ and $M_{\mathrm{Si}}$ points coincide with the $\bar{\Gamma}$ and $\bar{M}$ points of the repeated BZ of the reconstructed surface, respectively.
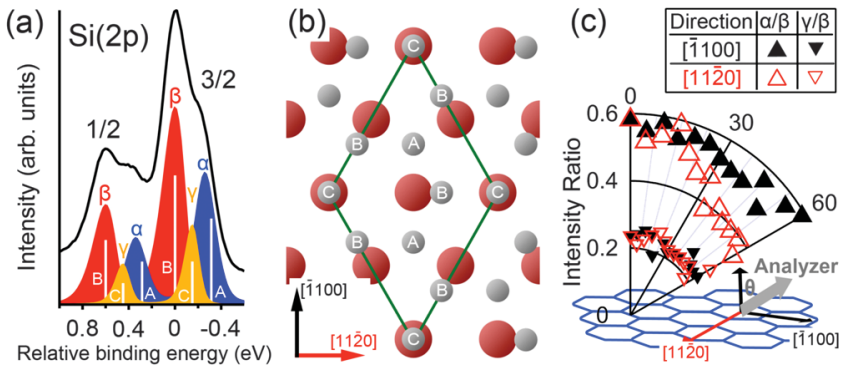

FIG. 2 (color). Chemical states and structural details of epitaxial Si layer on $\mathrm{ZrB}_{2}(0001)$. (a) Surface-sensitive Si $2 p$ photoelectron spectrum recorded at normal emission. Chemical states identified by a peak fitting procedure are labeled $\alpha, \beta$ and $\gamma$. The position of vertical lines $A, B$, and $C$ indicate the relative binding energies calculated for $\mathrm{Si}_{A}, \mathrm{Si}_{B}$, and $\mathrm{Si}_{C}$. The length of the lines is proportional to the number of chemically distinguishable atoms. (b) Model of the Si honeycomb structure on the topmost $\mathrm{Zr}$ layer of $\mathrm{ZrB}_{2}(0001)$. Chemically different types of $\mathrm{Si}$ atoms $\mathrm{Si}_{A}, \mathrm{Si}_{B}$ and $\mathrm{Si}_{C}$ are indicated together with the $(2 \times 2) \mathrm{UC}$ of $\mathrm{ZrB}_{2}(0001)$. (c) Intensity ratios $\alpha / \beta$ and $\gamma / \beta$ as a function of the polar photoelectron emission angle $\theta$, along the [1100] and [1120] directions of the $\mathrm{ZrB}_{2}(0001)$ thin film. 

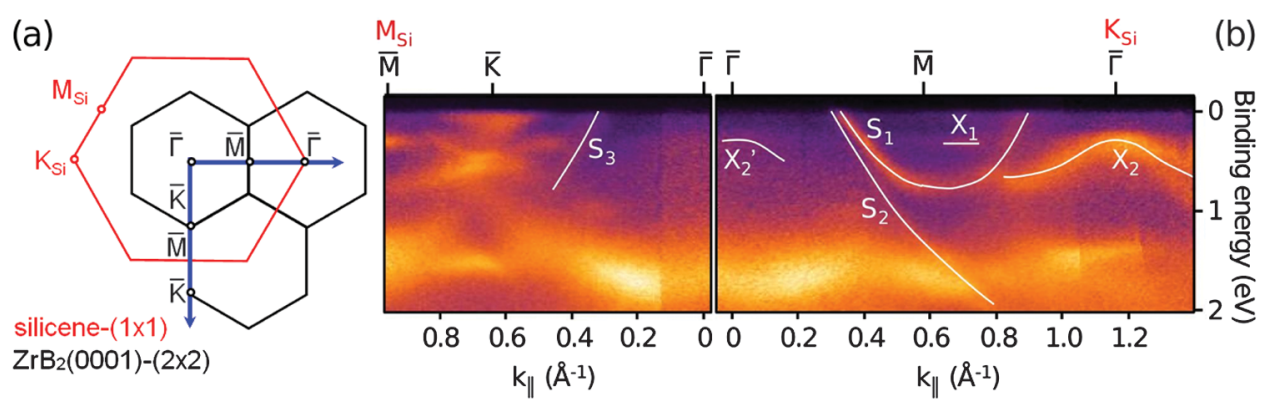

FIG. 3 (color). Experimental valence electronic structure of epitaxial Si layer on $\mathrm{ZrB}_{2}$ thin film. (a) BZs of the ( $\left.2 \times 2\right)$-reconstructed $\mathrm{ZrB}_{2}(0001)$ surface and of an unreconstructed silicene layer. (b) ARUPS spectra along the $\bar{\Gamma}-\bar{K}-\bar{M}$ and $\bar{\Gamma}-\bar{M}-\bar{\Gamma}$ high symmetry direction. Features $S_{n}$ and $X_{n}$ relate to surface electronic states of diboride and those of epitaxial Si layer, respectively.

ARUPS spectra along the $\bar{\Gamma}-\bar{K}-\bar{M}$ and $\bar{\Gamma}-\bar{M}-\bar{\Gamma}$ directions of the $(2 \times 2)$-reconstructed $\mathrm{ZrB}_{2}(0001)$ surface as a function of the in-plane electron wave number $k_{\|}$, are shown in Fig. 3(b) in form of intensity plots. Features denoted " $S_{1}$ " and " $S_{2}$ " as well as " $S_{3}$ " are related to the surface states characteristic for the $(1 \times 1) \mathrm{Zr}$-terminated $\mathrm{ZrB}_{2}$ single crystal [14,24]. Since the Zr-derived surface states are robust, the outermost $\mathrm{Zr}$ layer must be considered to be structurally intact. It is also consistent with only a minor degree of hybridization between the epitaxial Si layer and the mentioned diboride surface states since strong interactions between adsorbates and substrates modify or destroy surface electronic states [25]. Other features, denoted " $X_{1}$ ", " $X_{2}$ " and " $X_{2}^{\prime}$ ", which follow the symmetry of the $(2 \times 2)$ lattice, do not have a counterpart in the calculated band structure of the $\mathrm{ZrB}_{2}$ single crystal $[24,26]$ and are therefore related to the presence of the Si layer. The intense feature $X_{2}$ approaches the Fermi level by up to about $250 \mathrm{meV}$ at the $\bar{\Gamma}$ point of the repeated BZ and, owing to back-folding, is mirrored as $X_{2}^{\prime}$ with weak intensity in the 1st BZ. Note that the emission intensity pattern is typical for $\pi$ orbitals oriented in the direction normal to the surface [27]. The upward curvature of $X_{2}$ bears some resemblance to the predicted Dirac cone of $\pi$ bands of freestanding, nonreconstructed silicene at $K_{\mathrm{Si}}[1-4]$. While partial $s p^{3}$ hybridization caused by the buckling and interactions with the underlying metallic $\mathrm{ZrB}_{2}$ substrate are expected to occur, the existence of a $\pi$-electronic band structure itself proves that the bonding within the Si layer has a large degree of $s p^{2}$ character. This Si honeycomb structure may then be considered epitaxial silicene.

Buckling-induced band gap opening.-First-principles calculations of a silicene layer on a $\mathrm{ZrB}_{2}(0001)$ slab converge to two $\mathrm{Si}$ honeycomb structures with in-plane atom positions described in Fig. 2(b) and with different buckling. Their energy difference is $0.28 \mathrm{eV}$ per $\mathrm{Si}$ atom. The metastable structure depicted in Fig. 4(a) is consistent with the structural information obtained from the experiments. Note that strain relief at the boundaries of stress domains was not considered in the calculations, and may actually decrease the amount of buckling and affect the relative stability of possible structures. Within this structure, at a height of $z=2.124 \AA$ with respect to the topmost $\mathrm{Zr}$ layer, $\mathrm{Si}_{A}$ atoms on hollow sites occupy the lowest position, followed by $\mathrm{Si}_{C}$ atoms $(z=2.727 \AA)$ and $\mathrm{Si}_{B}$ atoms $(z=$ $3.026 \AA$ ). The calculated $\mathrm{Si}_{A}-\mathrm{Si}_{B}$ and $\mathrm{Si}_{B}-\mathrm{Si}_{C}$ bond lengths are $2.266 \AA$ and $2.242 \AA$, respectively, and as such match closely to the predicted bond lengths for freestanding honeycomb structures of $\mathrm{Si}(2.24-2.25 \AA)$ [1,3] which is noteworthy smaller than the bond length for purely $s p^{3}$-hybridized $\mathrm{Si}$ with diamond structure $(2.35 \AA)$. This is also in good agreement with average calculated nearest $\mathrm{Si}-\mathrm{Si}$ distance for silicene nanoribbon on $\operatorname{Ag}(110)(2.24 \AA)$ [10], in which the $\mathrm{Si}$ atoms are hybridized in between $s p^{2}$

(a)
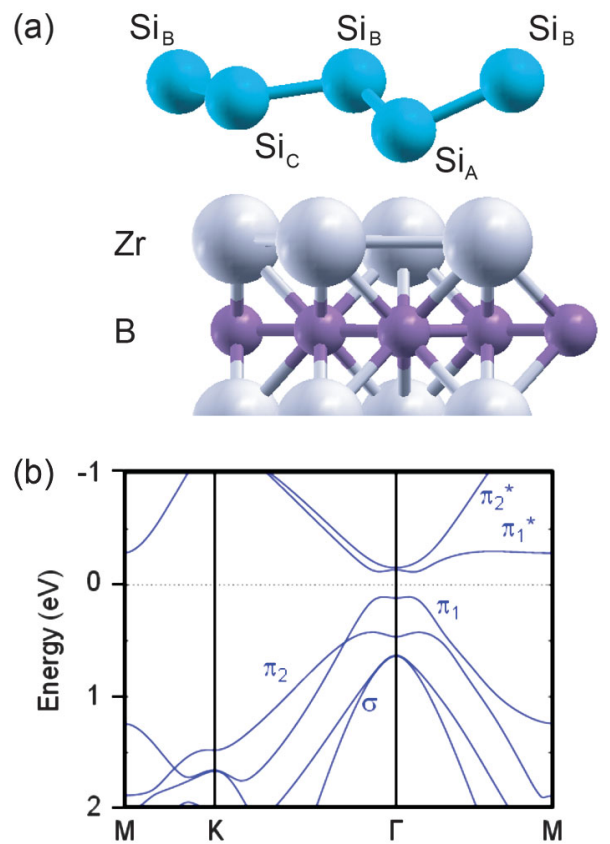

FIG. 4 (color). Calculated structure and band dispersion of epitaxial silicene. (a) Height profile of silicene on $\mathrm{Zr}$-terminated $\mathrm{ZrB}_{2}(0001)$. (b) Band structure of the freestanding, $(\sqrt{3} \times \sqrt{3})$ reconstructed silicene. High symmetry points correspond to those in $\mathrm{BZ}$ of $(2 \times 2)$-reconstructed $\mathrm{ZrB}_{2}(0001)$ shown in Fig. 3(a). 
and $s p^{3}$ [12]. The calculated bond angles $\mathrm{Si}_{B}-\mathrm{Si}_{C}-\mathrm{Si}_{B}$, $\mathrm{Si}_{B}-\mathrm{Si}_{A}-\mathrm{Si}_{B}, \mathrm{Si}_{A}-\mathrm{Si}_{B}-\mathrm{Si}_{A}$ and $\mathrm{Si}_{A}-\mathrm{Si}_{B}-\mathrm{Si}_{C}$ of $117.8^{\circ}$, $104.1^{\circ}, 104.9^{\circ}$, and $115.2^{\circ}$, respectively, indicate that the hybridization of $\mathrm{Si}$ atoms is varying from one atom to another. It seems that in order to accommodate the compressive epitaxial strain, a relatively large buckling as compared to that of freestanding silicene [3] is induced while the Si bond lengths are maintained to similar value.

As shown in Fig. 2(a), calculated shifts in initial-state core-level energies are in good agreement with the corelevel photoelectron spectrum. Atoms closer to the $\mathrm{Zr}$ plane are electron-rich, with energies related to $\mathrm{Si}_{A}$ and $\mathrm{Si}_{C}$ atoms shifted with respect to $\mathrm{Si}_{B}$ atoms by 315 and $150 \mathrm{meV}$, respectively. In order to understand if differences in the chemical environment of the $\mathrm{Si}$ atoms derive from structural properties of the silicene itself or are related to the electronic coupling to the substrate, these calculations have been repeated for a freestanding silicene layer maintaining the same internal structure which resulted in shifts of 160 and $20 \mathrm{meV}$, respectively. Since the order of relative positions of chemical states is unchanged, it is concluded that the existence of electron-rich or -poor atomic sites are primarily caused by the structure rather than by the coupling to the $\mathrm{ZrB}_{2}$ film. However, core-level energy shifts are larger for the coupled case, which indicates that interactions with the $\mathrm{ZrB}_{2}$ substrate play a significant role for the stabilization of the particular buckling.

Similarly, the gap opening may also originate from the structural properties of the layer itself, as demonstrated for rippled graphene [28], and/or from electronic coupling with the substrate, as shown for graphene on $\mathrm{SiC}$ [29]. To address if the particular atomistic buckling by itself can cause the opening of a gap, band structure calculations were carried out for a freestanding silicene layer with the structure shown in Fig. 4(a). The results plotted in Fig. 4(b) show a clear $\pi$ band structure with a band gap in agreement with the features $X_{2}$ and $X_{2}^{\prime}$ in Fig. 3(b). The calculation verifies that the opening of a $\pi$-electronic gap results primarily from the particular buckling. Additionally, the presence of $\pi$ bands proves $s p^{2}$ hybridization even for this relatively large degree of buckling. Given the relatively small experimental band width of feature $X_{2}$, it may possibly be conceived that feature $X_{1}$ in Fig. 3(b) is also related to a state involving silicene $\pi$ orbitals. Obviously, in future work, it is then important to elucidate the role of silicene-diboride interactions for electronic properties like the splitting and dispersion of $\pi$-electronic bands, and also in the formation process of epitaxial silicene.

In summary, the combination of experimental and theoretical data provides evidence for the presence of buckled, epitaxial silicene on the diboride surface. The buckling induced by epitaxy determines the electronic properties that are different from those so far predicted for freestanding silicene. These results imply that the atomistic structure and thus the electronic properties of silicene can be tuned by the application of an external stress. This can be realized, for instance, through epitaxial growth on an appropriate substrate with selected lattice parameter. The results show that $(\sqrt{3} \times \sqrt{3})$-reconstructed silicene is so far the only crystalline silicon allotrope with a direct $\pi$-electronic band gap. This is likely to be fundamentally relevant for future applications of silicene in optoelectronic and transistor applications, in particular, and for the miniaturization of Si-based electronics in general.

We are grateful to experimental help from A. Harasawa and Dr. K. Yaji (The University of Tokyo). Part of this work has been performed under the approval of the Photon Factory Program Advisory Committee (Proposal No. 2010G571). This research was supported by Special Coordination Funds for Promoting Science and Technology commissioned by MEXT, Japan; KAKENHI (No. 22560006, No. 22015008), and also by Funding Program for Next Generation World-Leading Researchers (GR046). A. F. acknowledges a fellowship from the Japan Society for the Promotion of Science, and Y.W. from the MARUBUN Foundation.

Note added.-Recently, new results for silicene sheets on $\operatorname{Ag}(111)$ were published [30-32].

*Author to whom correspondence should be addressed. yukikoyt@jaist.ac.jp.

[1] K. Takeda and K. Shiraishi, Phys. Rev. B 50, 14916 (1994).

[2] G. G. Guzmán-Verri and L. C. Lew Yan Voon, Phys. Rev. B 76, 075131 (2007).

[3] S. Cahangirov, M. Topsakal, E. Aktürk, H. Sahin, and S. Ciraci, Phys. Rev. Lett. 102, 236804 (2009).

[4] M. Houssa, G. Pourtois, V. V. Afanas'ev, and A. Stesmans, Appl. Phys. Lett. 97, 112106 (2010).

[5] R. Baptist, S. Ferrer, G. Grenet, and H. C. Poon, Phys. Rev. Lett. 64, 311 (1990).

[6] T. Hirano and J. Fujiwara, Phys. Rev. B 43, 7442 (1991).

[7] P. Wetzel et al., Solid State Commun. 82, 235 (1992).

[8] P. Wetzel, S. Saintenoy, C. Pirri, D. Bolmont, and G. Gewinner, Phys. Rev. B 50, 10886 (1994).

[9] P. De Padova et al., Nano Lett. 8, 271 (2008).

[10] B. Aufray et al., Appl. Phys. Lett. 96, 183102 (2010).

[11] P. De Padova et al., Appl. Phys. Lett. 96, 261905 (2010).

[12] P. De Padova, C. Quaresima, B. Olivieri, P. Perfetti, and G. Le Lay, Appl. Phys. Lett. 98, 081909 (2011).

[13] B. Lalmi et al., Appl. Phys. Lett. 97, 223109 (2010).

[14] Y. Yamada-Takamura, F. Bussolotti, A. Fleurence, S. Bera, and R. Friedlein, Appl. Phys. Lett. 97, 073109 (2010).

[15] Y. Yamada-Takamura et al., Phys. Rev. Lett. 95, 266105 (2005).

[16] R. Friedlein et al., Phys. Rev. B 68, 195414 (2003).

[17] J. P. Perdew, K. Burke, and M. Ernzerhof, Phys. Rev. Lett. 77, 3865 (1996). 
[18] T. Ozaki, Phys. Rev. B 67, 155108 (2003).

[19] T. Ozaki et al., http://www.openmx-square.org/.

[20] O. L. Alerhand, D. Vanderbilt, R. D. Meade, and J.D. Joannopoulos, Phys. Rev. Lett. 61, 1973 (1988).

[21] C. J. Karlsson, E. Landemark, Y.-C. Chao, and R. I. G. Uhrberg, Phys. Rev. B 50, 5767 (1994).

[22] After subtraction of a Shirley background, peaks were fitted with a branch ratio of 2 and a spin-orbit split of $600 \pm 10 \mathrm{meV}$. The $\alpha, \beta$, and $\gamma$ peak doublets were fitted with asymmetric parameters of $0,0.07$ and 0.14 , Gauss/Lorentzian ratios of $0.7,0.95$, and 0.7 and fullwidths at half maximum of 220, 230, and $150 \mathrm{meV}$, respectively.

[23] E. L. Bullock et al., Phys. Rev. Lett. 74, 2756 (1995).
[24] S. Kumashiro et al., e-J. Surf. Sci. Nanotech. 4, 100 (2006).

[25] C. H. Schwalb et al., Phys. Rev. Lett. 101, 146801 (2008).

[26] T. Aizawa, W. Hayami, and S. Otani, Phys. Rev. B 65, 024303 (2001).

[27] F. Bussolotti, S. W. Han, Y. Honda and R. Friedlein, Phys. Rev. B 79, 245410 (2009).

[28] M. Papagno et al., ACS Nano 6, 199 (2012).

[29] S. Y. Zhou et al., Nature Mater. 6, 770 (2007).

[30] C.-L. Lin et al., Appl. Phys. Express 5, 045802 (2012).

[31] H. Jamgotchian et al., J. Phys. Condens. Matter 24, 172001 (2012).

[32] P. Vogt et al., Phys. Rev. Lett. 108, 155501 (2012). 